\section{A novel homozygous variation in the PANK2 gene in two Persian siblings with atypical pantothenate kinase associated neurodegeneration}

\author{
Amir Hasan Habibi, ${ }^{1}$ Saeed Razmeh, 2 \\ Omid Aryani, ${ }^{1}$ Mohammad Rohani, ${ }^{1}$ \\ Laleh Taghavian, ${ }^{2}$ Elham Alizadeh, ${ }^{2}$ \\ Karim Moradian Kokhedan² \\ Maryam Zaribafian ${ }^{1}$ \\ 1 Iran University of Medical Sciences, \\ Tehran; ${ }^{2}$ Yasuj University of Medical \\ Sciences, Yasuj, Iran
}

\begin{abstract}
Pantothenate Kinase-associated Neurodegeneration (PKAN) is an autosomal recessive disorder that is caused by variation in pantothenate kinase-2 gene (PANK2) gene on chromosome 20. The common presentation of this disease includes progressive dystonia, Parkinsonism, retinopathy, cognitive impairment, and spasticity. The typical magnetic resonance imaging finding is eye of the tiger sign in globus pallidus and not pathogenic and not found in all patients. In the present study, we describe two siblings who have a novel variation of the PANK2 gene. These patients with the same genotype, have different ages at the onset of disease and also the various severity of the disease. The description of these cases helps to understand this disease, its symptoms, pathogenesis, and its treatment.
\end{abstract}

\section{Introduction}

Neurodegeneration with brain iron accumulation (NBIA) includes a heterogeneous group of hereditary neurodegenerative diseases that occur with symptoms such as dystonia, parkinsonism, cognitive impairment and vision loss. ${ }^{1-3}$ The most common type is Pantothenate Kinase-associated Neurodegeneration (PKAN), due to a variation in the pantothenate kinase-2 gene (PANK2) gene on chromosome 20.4 The disease has two types, classic and atypical, the classical type occurs at an earlier age and more severe. The typical MRI finding is eye of the tiger sign that is T2-hypointensity surrounding a central hyperintensity in globus pallidus and not pathogenic and not found in all patients. $^{5-7}$ In this paper, we present two siblings of patients with PKAN who have a novel variation of the $P A N K 2$ gene.

\section{Case Report}

A 32-year-old woman (case 1) was referred to our clinic because of gait difficulties that had started at the age of about 16 years. The family history, drug history, and past medical history were noncontributory. On neurologic examination, the Mini-Mental State Examination (MMSE) was normal. Cranial nerve examination was normal and the Ophthalmologic evaluation indicated no evidence of visual impairment, KayserFleischer rings, pigmentary retinopathy and atrophy of optic nerve. She had hypokinesia and bradykinesia and limb rigidity, which was more on the right side, also there were generalized dystonia and spasticity of lower limbs without cerebellar signs. Acanthocytosis was not seen in the peripheral blood smear and the other laboratory tests were normal. A brain magnetic resonance image (MRI) revealed the eye of the tiger sign (Figure 1A), and the genetic analysis showed a homozygous variation in the PANK2 gene NM_153638.3:c.706_708delGAA (GAA deletion, p.E236 Del).

The second patient (case 2) was a 30 year-old man presents with generalized dystonia and Parkinsonism that had started at the age of about 18 years. Gradually, the dystonia increased in severity, His developmental history was normal, the ophthalmological examination was normal with no evidence of visual impairment and retinopathy. He also had a generalized dystonia, hypokinesia, and limb rigidity. There were no pyramidal or cerebellar signs. Brain MRI discloses a typical eye-ofthe-tiger sign (Figure 1B), and a genetic study detected a pathogenic variation in the PANK2 gene (GAA deletion, p.E236 Del). For DNA extraction from type of blood cells, Q1AAMP DNA MICROKIT (Cat number: 56304) was used. For comprehensive investigation of all the exons and splicing sites of the introns in PANK2 gene, primers from another paper were used (Table 1). ${ }^{8}$ Briefly, PCR reaction was performed in a final volume of $25 \mu \mathrm{L}$ containing 100-200 ng of total DNA, 10 pmol of each primer, $2.5 \mathrm{mM} \mathrm{MgCl} 2,200$ $\mathrm{mM}$ each of dNTP and $1 \mathrm{U}$ Taq DNA polymerase (Roche Diagnostics, Mannheim, Germany). The reaction mixture was cycled 35 times at $95^{\circ} \mathrm{C}$ for 1 min, annealing temperature $\left({ }^{\circ} \mathrm{C}\right)$ for $1 \mathrm{~min}$ (refer to Table 1) and $72{ }^{\circ} \mathrm{C}$ for $1 \mathrm{~min}$. Electrophoresis of the PCR products was
Correspondence: Saeed Razmeh, Yasuj University of Medical Sciences, Kohgiluyeh and Boyer-Ahmad Province, Yasuj, Shahid Motahari Blvd, Iran.

E-mail: srazmeh@yahoo.com

Key words: Pantothenate Kinase-associated Neurodegeneration, novel variation, pantothenate kinase-2 gene.

Acknowledgements: The authors thank Dr. magid kheradmand maher for typing and editing the manuscript.

Contributions: the authors contributed equally.

Conflict of interest: the authors declare no potential conflict of interest.

Funding: none.

Received for publication: 30 November 2018 Accepted for publication: 14 January 2019.

This work is licensed under a Creative Commons Attribution NonCommercial 4.0 License (CC BY-NC 4.0).

(C) Copyright A.H. Habibi et al., 2019

Licensee PAGEPress, Italy

Neurology International 2019; 11:7959

doi:10.4081/ni.2019.7959

performed on $2 \%$ agarose gels (Figure 2). The PCR products were sequenced with the forward or reversed primers on an $\mathrm{ABI}$ 3730XL sequencer (Macrogen Company, Korea) and compared with control samples using the FinchTV program and analyzed on the NCBI website (http://blast.ncbi.nlm.nih.gov/Blast.cgi).Wit $\mathrm{h}$ these methods, the target sequence for each patient was compared with the normal reference sequence, and mutations in the exons and the splicing sites of the introns in the PANK2 gene. All transcribed exons of the PANK2 gene were investigated in patients. delGAA homozygous mutation in the exon 2 was detected in both siblings and their parent were heterozygous in this location. Both patients were treated with Madopar 125 three times daily and Deferiprone 500 twice daily, which we did not see any changes in symptoms after a three month follow up.

We obtained the written informed consent from the two patients for publication of the case report

\section{Discussion and Conclusions}

Pantothenate

Kinase-Associated Neurodegeneration (PKAN) is an autosomal recessive disorder caused by a 
variation in the PANK2 gene. The clinical manifestations include speech disorder Parkinsonism, dystonia, spasticity, Visual loss, dysphagia seizure, and dementia.2,3,9 All of the patients with Pantothenate Kinase Associated Neurodegeneration do not have eye-of-the-tiger sign especially in the early stages of this disease and all of the patients with this sign do not have a PANK2 mutation. ${ }^{10-13}$ This gene has seven exons, most variations are missense but duplication, deletion and splice also reported, $6,14,15$ in our patients, there is a novel variation that was GAA deletion resulting in the p.E236del that bioinformatically or in silico showed it is probably pathogenic mutation but needs further investigation. Two of our patients had manifestations but the progression, and severity of the disease was different. The course of the disease in the first patient was faster and the symptoms were more severe.

Case 2 had a later onset of symptoms, about 18 years, with slow progression and the severity of the symptoms was milder. Usually Bulbar symptoms, generalized dystonia, and gait disturbance, are more common in classical form and tremors, segmental dystonia, seizure, psychiatric disorders and Parkinsonism more common in atypical form of PKAN disease.4,16,17 Because the precise mechanism of these diseases has not been discovered so far, most of the treatments used so far reduce the severity of symptoms to some extent.1,18 The dystonia is debilitating in these patients and is exacerbated over time and is treated with drugs such as Oral and Intrathecal Baclofen, Botulinum Toxin, gabapentin and anticholinergics. Surgery such as Deep brain stimulation, ablative pallidotomy or thalamotomy is also used to treat this disease. ${ }^{18-21}$ Iron chelator drugs such as deferiprone have been used in some studies in neurodegenerative diseases and have promising results. This drug passes through the blood-

Table 1. Primers for studying the $P A N K 2$ gene by PCR-sequencing.

\begin{tabular}{|c|c|c|c|}
\hline Primers & Forward/Reverse & Product size (bp) & $\mathrm{TM}\left({ }^{\circ} \mathrm{C}\right)$ \\
\hline 1 & $\begin{array}{l}\text { F: GGAACTAGGCCGAGGGACAAAG } \\
\text { R:GAAGGTACCGCTTTCGGAG }\end{array}$ & 1200 & 63 \\
\hline 2 & $\begin{array}{c}\text { F: GCCCCAAAACCCTTTTGC } \\
\text { R: ACCACCTCTAGATGGCCAACTC }\end{array}$ & 650 & 60 \\
\hline 3 & $\begin{array}{c}\text { F: TGGGTCTGTAGTAGCAGG } \\
\text { R: CATTTGTTTGCATAATCCAG }\end{array}$ & 580 & 54 \\
\hline 4 & $\begin{array}{l}\text { F: GACATGGGCCCTGTGTTTTG } \\
\text { R: GGCCCGCCTGTATTTCTTAG }\end{array}$ & 438 & 60 \\
\hline 5 & $\begin{array}{l}\text { F: GCTCTGTTTGAAGTTTGC } \\
\text { R:ATGACTACATTTAGGCACTG }\end{array}$ & 402 & 54 \\
\hline 6 & $\begin{array}{c}\text { F: TCCTGTGACATTATCTAGCATG } \\
\text { R: AGCCCATTTCACCTCCAC }\end{array}$ & 429 & 54 \\
\hline 7 & $\begin{array}{l}\text { F:TCTGAAGTGCTTGGATAGTC } \\
\text { R:CTTCCTGGTTGCTAATTTAG }\end{array}$ & 454 & 54 \\
\hline
\end{tabular}
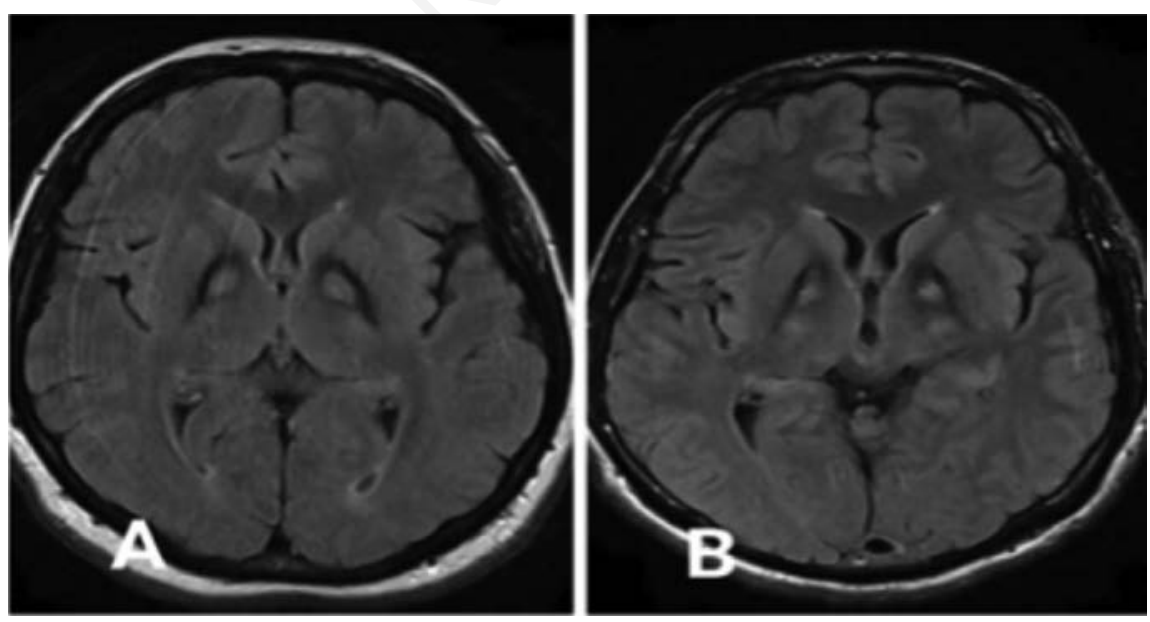

Figure 1. Brain magnetic resonance imaging T2-FLAIR images of case 1 (A) and 2 (B) revealed the eye of the tiger sign. brain barrier and reduces brain iron and reduces the oxidative stress response in the brain. ${ }^{21-24}$ In conclusion, although the disease is rare. But familiarity with clinical and radiological symptoms can help to diagnose this disease more easily.

\section{References}

1. Gregory A, Polster BJ, Hayflick SJ. Clinical and genetic delineation of neurodegeneration with brain iron accumulation. J Med Genet 2009;46:73-80.

2. Schipper HM. Neurodegeneration with brain iron accumulation: clinical syndromes and neuroimaging. Biochim Biophys Acta 2012;1822:350-60.

3. Schneider SA, Hardy J, Bhatia KP. Syndromes of neurodegeneration with brain iron accumulation (NBIA): an update on clinical presentations, histological and genetic underpinnings, and treatment considerations. Mov Disord 2012;27:42-53.

4. Pellecchia M, Valente E, Cif L, et al. The diverse phenotype and genotype of pantothenate kinase-associated neurodegeneration. Neurology 2005;64: 1810-2.

5. Gregory A, Hayflick SJ. Pantothenate kinase-associated neurodegeneration. 2013. Seattle (WA): University of Washington, Seattle.

6. Parmar A, Khare S, Srivastav V. Pantothenate-kinase associated neurodegeneration. J Assoc Physicians India 2012;60:74-6.

7. Zorzi G, Zibordi F, Chiapparini L, et al. Iron-related MRI images in patients with pantothenate kinase-associated neurodegeneration (PKAN) treated with deferiprone: Results of a phase II pilot trial. Mov Disord 2011;26:1755-9.

8. Aryani O, Houshmand M, Fatehi F. A novel PANK2 gene mutation in a Persian boy: the first report from Iran. Clin Neurol Neurosurg 2013;115:1170.

9. Razmeh S, Habibi AH, Orooji M, et al. Pantothenate kinase-associated neurodegeneration: Clinical aspects, diagnosis and treatments. Neurol Intern 2018;10.

10. Chiapparini L, Savoiardo M, D’Arrigo $\mathrm{S}$, et al. The "eye-of-the-tiger" sign may be absent in the early stages of classic pantothenate kinase associated neurodegeneration. Neuropediatrics 2011;42:159-62.

11. Hájek M, Adamovičová M, Herynek V, et al. MR relaxometry and $1 \mathrm{H}$ MR spectroscopy for the determination of iron and metabolite concentrations in PKAN patients. Eur Radiol 2005;15:1060-8. 
12. Hayflick S, Hartman M, Coryell J, et al. Brain MRI in neurodegeneration with brain iron accumulation with and without PANK2 mutations. Am J Neuroradiol 2006;27:1230-3.

13. Kumar N, Boes CJ, BabovicVuksanovic D, Boeve BF. The "eye-ofthe-tiger" sign is not pathognomonic of the PANK2 mutation. Arch Neurol 2006;63:292.

14. Hartig M, Prokisch H, Meitinger T, Klopstock T. Pantothenate kinase-associated neurodegeneration. Curr Drug Targets 2012;13:1182-9.

15. Lee C-H, Lu C-S, Chuang W-L, et al. Phenotypes and genotypes of patients with pantothenate kinase-associated neurodegeneration in Asian and Caucasian populations: 2 cases and literature review. Sci World J 2013;2013.

16. Lee J-H, Park J, Ryu H-S, et al. Clinical heterogeneity of atypical pantothenate kinase-associated neurodegeneration in
Koreans. J Mov Disord 2016;9:20.

17. Marelli C, Piacentini S, Garavaglia B, et al. Clinical and neuropsychological correlates in two brothers with pantothenate kinase-associated neurodegeneration. Mov Disord 2005;20:20812.

18. Pratini NR, Sweeters N, Vichinsky E, Neufeld JA. Treatment of Classic Pantothenate Kinase-Associated Neurodegeneration (PKAN) with Deferiprone and Intrathecal Baclofen. Am J Phys Med Rehabil 2013;92:728.

19. Castelnau P, Cif L, Valente EM, et al. Pallidal stimulation improves pantothenate kinase-associated neurodegeneration. Ann Neurol 2005;57:73841.

20. Mikati MA, Yehya A, Darwish H, et al. Deep brain stimulation as a mode of treatment of early onset pantothenate kinase-associated neurodegeneration. Eur J Paediatr Neurol 2009;13:61-4.
21. Rohani M, Razmeh S, Shahidi GA, Orooji M. A pilot trial of deferiprone in pantothenate kinase-associated neurodegeneration patients. Neurol Int 2018;9:7279.

22. Cossu G, Abbruzzese G, Matta G, et al. Efficacy and safety of deferiprone for the treatment of pantothenate kinaseassociated neurodegeneration (PKAN) and neurodegeneration with brain iron accumulation (NBIA): results from a four years follow-up. Parkinson Relat Disord 2014;20:651-4.

23. Rouault TA. Iron metabolism in the CNS: implications for neurodegenerative diseases. Nat Rev Neurosci 2013;14:551.

24. Szumowski J, Bas E, Gaarder K, et al. Measurement of brain iron distribution in Hallevorden-Spatz syndrome. J Magn Res Imag 2010;31:482-9. 